\title{
1 Four-quadrant Silicon and Silicon Carbide 2 Photodiodes for Beam Position Monitor 3 Applications: Electrical Characterization and 4 Electron Irradiation Effects
}

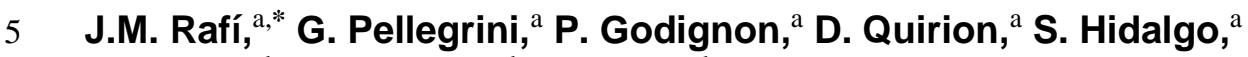 \\ 6 O. Matilla, ${ }^{\mathrm{b}}$ A. Fontserè, ${ }^{\mathrm{b}}$ B. Molas, ${ }^{\mathrm{b}}$ K. Takakura, ${ }^{\mathrm{c}}{ }^{\text {I. Tsunoda, }},{ }^{\mathrm{c}}$ M. Yoneoka, ${ }^{\mathrm{c}}$ \\ 7 D. Pothin ${ }^{\mathrm{d}}$ and P. Fajardo ${ }^{\mathrm{d}}$ \\ $8{ }^{a}$ Instituto de Microelectrónica de Barcelona, CNM-CSIC, Campus UAB, 08193 Bellaterra, Spain \\ $9 \quad{ }^{b}$ ALBA Synchrotron, Carrer de la Llum 2-26, 08290 Cerdanyola del Vallès, Spain \\ 10 \\ ${ }^{c}$ National Institute of Technology, Kumamoto College, 2659-2 Suya, Kumamoto 861-1102, Japan \\ ${ }^{d}$ ESRF The European Synchrotron, 71 Avenue des Martyrs, 38000 Grenoble, France \\ E-mail: jm.rafiecsic.es
}

ABSTRACT: Silicon photodiodes are very useful devices as X-ray beam monitors in synchrotron radiation beamlines, as well as other astronomy and space applications. Owing to their lower susceptibility to variable temperature and illumination conditions, there is also special interest in silicon carbide devices for some of these applications. Moreover, radiation hardness of the involved technologies is a major concern for high-energy physics and space applications. This work presents four-quadrant photodiodes produced on ultrathin $(10 \mu \mathrm{m})$ and bulk Si, as well as on $\mathrm{SiC}$ epilayer substrates. An extensive electrical characterization has been carried out by using current-voltage $(\mathrm{I}-\mathrm{V})$ and capacitance-voltage $(\mathrm{C}-\mathrm{V})$ techniques. The impact of different temperature (from $-50{ }^{\circ} \mathrm{C}$ to $175^{\circ} \mathrm{C}$ ) and visible light conditions on the electrical characteristics of the devices has been evaluated. Radiation effects caused by $2 \mathrm{MeV}$ electron irradiation up to $1 \times 10^{14}, 1 \times 10^{15}$ and $1 \times 10^{16} \mathrm{e} / \mathrm{cm}^{2}$ fluences have been studied. Special attention has been devoted to the study of charge build-up in diode interquadrant isolation, as well as its impact on interquadrant resistance. The study of these electrical properties and its radiation-induced degradation should be taken into account for device applications.

KEYWORDS: Beam-line instrumentation (beam position and profile monitors; beam-intensity monitors; bunch length monitors); X-ray detectors; Si microstrip and pad detectors; Radiation damage to detector materials (solid state).

* Corresponding author. 
$\begin{array}{ll}\text { 4. Electron irradiation effects } & 7\end{array}$

5. Summary 9

\section{Introduction}

Silicon photodiodes are very useful devices as X-ray beam monitors in synchrotron radiation beamlines. In order to be used in transmissive mode and given the absorption properties of silicon, the devices must be thinner than $10 \mu \mathrm{m}$ to achieve X-ray transmission higher than $90 \%$ for photon energies above $10 \mathrm{keV}$ [1-3]. On the other hand, bulk silicon segmented devices are also of interest for astronomy and space applications, such as solar tracking systems [4]. Owing to their lower susceptibility to variable temperature and illumination conditions, there is also special interest in silicon carbide devices for some of these applications [5,6]. Superior radiation resistance of $\mathrm{SiC}$ compared to $\mathrm{Si}$ was anticipated in some early works and this was attributed to its higher atomic displacement threshold energy [7,8]. However, the existence of different polytypes and difficulties in crystal growth have often made this difficult to assess [9,10]. Very high radiation dose rates, in the range of $1 \mathrm{Mrad} / \mathrm{s}$, are easily reached in synchrotron beams, however, the X-rays maximum energy transfer to $\mathrm{Si}$ or $\mathrm{SiC}$ atoms is below the threshold energy for radiation-induced dislocation of the crystalline lattice and therefore no bulk damage is expected [11]. Nevertheless, the devices may still degrade owing to generation and trapping of charge in dielectric layers used in their isolation and passivation, as well as surface currents associated with radiation-induced interface traps [12]. The study of radiation effects on the involved technologies is also of special interest for high-energy physics and space applications.

In this work, four-quadrant photodiodes produced on ultrathin $(10 \mu \mathrm{m})$ and bulk $\mathrm{Si}$, as well as on $\mathrm{SiC}$ epilayer substrates are studied. The devices have been fabricated with different design parameters along with auxiliary technology test structures (single diodes and MOS capacitors). Electrical characterization has been carried out by using current-voltage (I-V) and capacitancevoltage $(\mathrm{C}-\mathrm{V})$ techniques. The impact of different temperature, from $-50{ }^{\circ} \mathrm{C}$ to $175{ }^{\circ} \mathrm{C}$, and visible light conditions on the electrical characteristics of the devices has been evaluated. Finally, the effects of $2 \mathrm{MeV}$ electron irradiation, up to $1 \times 10^{14}, 1 \times 10^{15}$ and $1 \times 10^{16} \mathrm{e} / \mathrm{cm}^{2}$ fluences, have been also studied. 


\section{Four quadrant diodes fabrication}

Device fabrication is based on IMB-CNM p-on-n diode processing experience on both high resistivity silicon [13] and silicon carbide substrates [6].

(a)
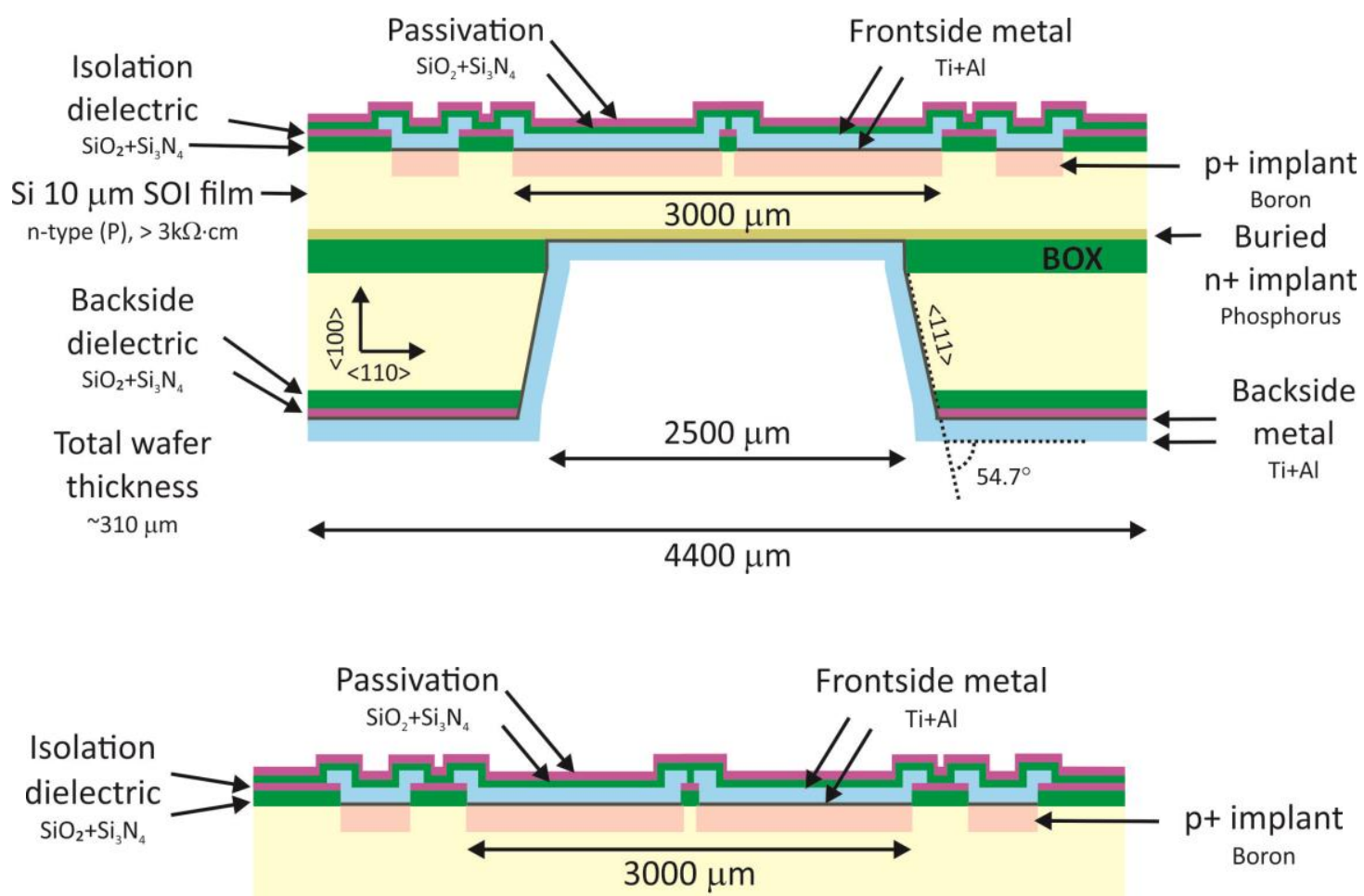

(b)

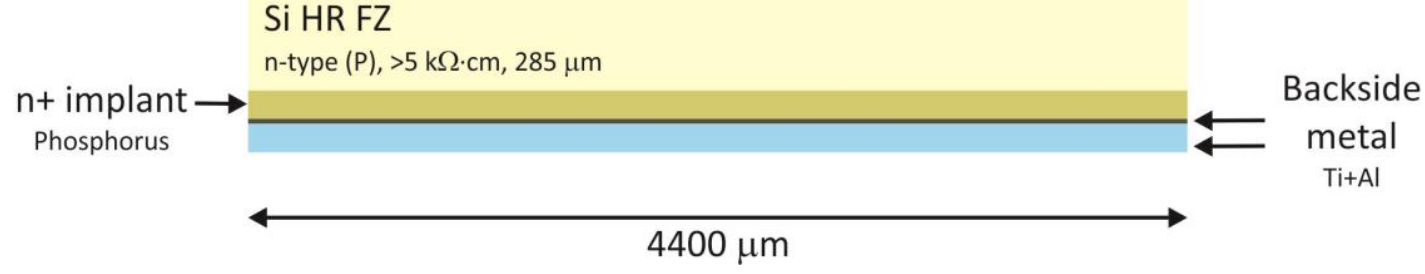

(c)

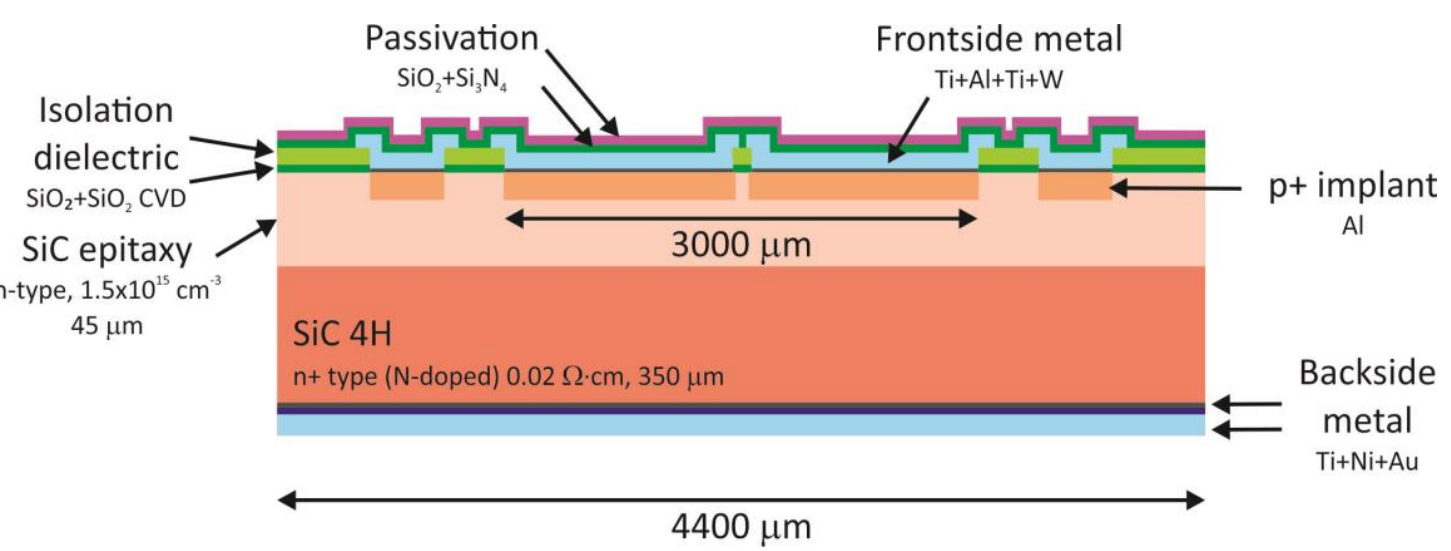

Figure 1. Schematic cross sections (not drawn to scale) of a four-quadrant diode with its guard ring fabricated on (a) ultrathin $(10 \mu \mathrm{m})$ Si layer, (b) bulk Si and (c) epitaxied SiC substrates. 


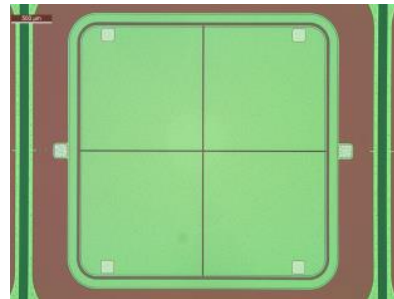

(a)

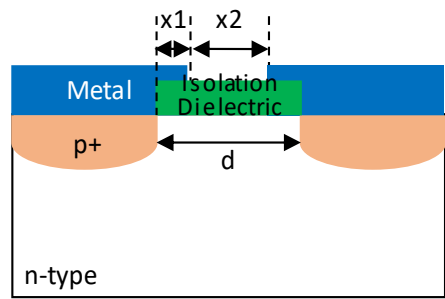

(b)

\begin{tabular}{|c|c|c|c|}
\hline $\begin{array}{c}\text { Device } \\
\text { type }\end{array}$ & $\begin{array}{c}\mathrm{d} \\
{[\mu \mathrm{m}]}\end{array}$ & $\begin{array}{c}\mathrm{x}_{1} \\
{[\mu \mathrm{m}]}\end{array}$ & $\begin{array}{c}\mathrm{x}_{2} \\
{[\mu \mathrm{m}]}\end{array}$ \\
\hline Single & 0 & - & - \\
\hline 4Q-A & 10 & 3 & 4 \\
\hline 4Q-B & 15 & 3 & 9 \\
\hline 4Q-C & 25 & 5 & 15 \\
\hline
\end{tabular}

(c)
100

101

102

103
Figure 2. (a) Optical microscopy picture of a fabricated four-quadrant diode, (b) sketch with the definition of the interquadrant distance " $\mathrm{d}$ ", as well as the metal overlapped and non-overlapped isolation regions ( $\mathrm{x}_{1}$ and $\mathrm{x}_{2}$, respectively), (c) device types used in the present work.
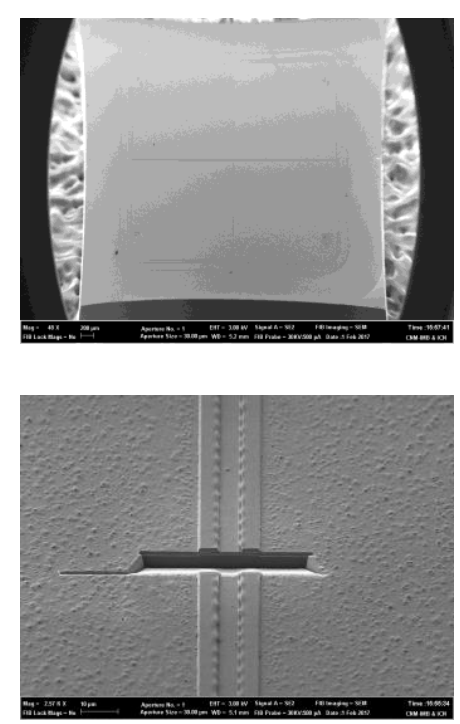
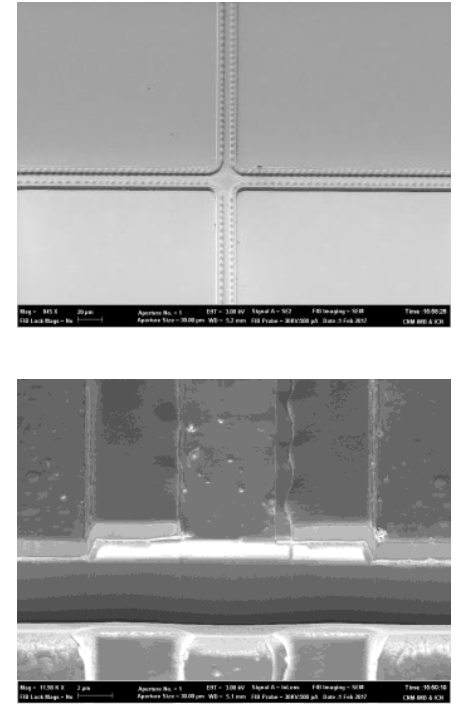
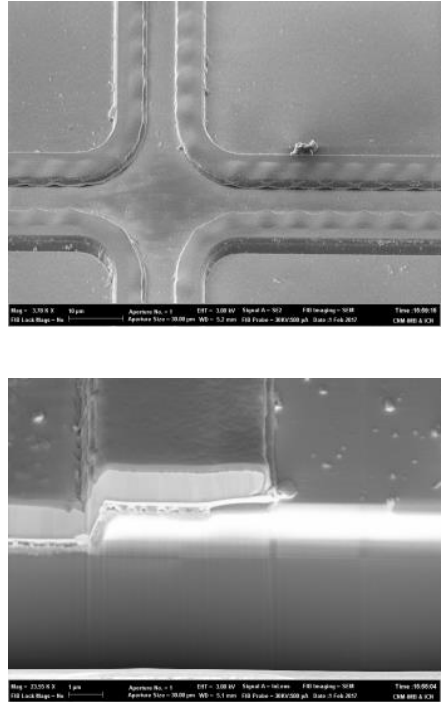

Figure 3. SEM pictures of a SiC four-quadrant diode (4Q-A type) subjected to ion milling with 
Post-fabrication inspection of the devices on the different substrates was carried out by means of Optical Microscopy, Scanning Electron Microscopy (SEM), Energy Dispersive X-ray Spectroscopy (EDX) and Focused Ion Beam (FIB) techniques. Figure 3 shows SEM pictures of a $\mathrm{SiC}$ four-quadrant diode subjected to FIB ion milling in a localized area across the diode interquadrant region.

\section{Electrical characterization of non-irradiated devices}

Current-voltage (I-V) and Capacitance-Voltage (C-V) measurements were carried out in a Summit 11000B-M light-proof and electrically shielded probe station by using an HP 4155B semiconductor parameter analyzer and an Agilent 4284A Precision LCR Meter.

\subsection{Temperature effects}

In order to evaluate the electrical characteristics of the devices fabricated on the different substrates, diode I-V curves were measured at different temperatures by using an Espec ETC200L thermal system with the wafer prober thermal chuck. The I-V characteristics were measured with three independent HP 4155B source monitor units (SMUs) connected to the diode, ring and backside terminals. While zero potential was applied to the diode and ring terminals, a voltage sweep was performed on the backside terminal.

Figure 4(a) shows the current density measured for bulk Si, $10 \mu \mathrm{m}$-thick $\mathrm{Si}$ and epilayer $\mathrm{SiC}$ single diodes at four different temperatures $\left(-50^{\circ} \mathrm{C}, 25^{\circ} \mathrm{C}, 100{ }^{\circ} \mathrm{C}\right.$ and $\left.175^{\circ} \mathrm{C}\right)$. From the figure, a clear increase in both, reverse and forward bias current levels is observed on bulk $\mathrm{Si}$ and $10 \mu \mathrm{m}$-thick Si diodes when increasing measurement temperature. Lower conduction levels are observed for the 10- $\mu \mathrm{m}$ thick Si diodes compared to their thicker bulk Si counterparts. In particular, the reverse leakage current at $-50^{\circ} \mathrm{C}$ for the thin Si diodes is found to be in the range of the experimental set-up resolution $(\sim \mathrm{pA})$. For what concerns the reverse bias breakdown voltage, all devices have been tested up to $100 \mathrm{~V}$, and only for the $10-\mu \mathrm{m}$ thick Si diodes breakdown has been observed at $40 \mathrm{~V}$. Interestingly for potential applications, no significant increase is observed for the reverse current in $\mathrm{SiC}$ diodes when increasing the measurement temperature, this can be attributed to its wider bandgap energy (3.2 eV) compared to silicon (1.1 $\mathrm{eV})$, reducing the thermal generation of carriers. Only some increase in forward bias current levels is observed, what can be indeed regarded as a diode threshold voltage lowering. This is due to junction built-in potential decrease as a result of semiconductor intrinsic carrier concentration increase with temperature. Finally, the observed forward bias current density saturation corresponds to a fixed $1 \times 10^{-3}$ A SMU current compliance.

The reverse current dependence on temperature can be better appreciated in Fig. 4(b), where the current density values corresponding to some fixed reverse bias conditions ( $2 \mathrm{~V}$ for bulk $\mathrm{Si}$ and $10 \mu \mathrm{m}$-thick $\mathrm{Si}$ devices and $10 \mathrm{~V}$ for epitaxied SiC diodes) have been plotted against measuring temperature. From Fig. 4(b), Arrhenius-law dependences can be drawn for Si devices, with corresponding activation energies about $0.56 \mathrm{eV}$ and $0.66 \mathrm{eV}$ for the bulk Si and $10 \mu \mathrm{m}$-thick Si devices, respectively. These values about half of the silicon bandgap energy point to generation current as the dominant contribution to the reverse current, especially for the bulk $\mathrm{Si}$ devices. On the other hand, the slightly higher activation energy extracted for the 10 $\mu$ m-thick Si devices may suggest some slight contribution of diffusion mechanism in these thinner devices [15]. In contrast, under the studied measurement conditions, no appreciable temperature dependence is observed for the $\mathrm{SiC}$ diodes reverse current. 


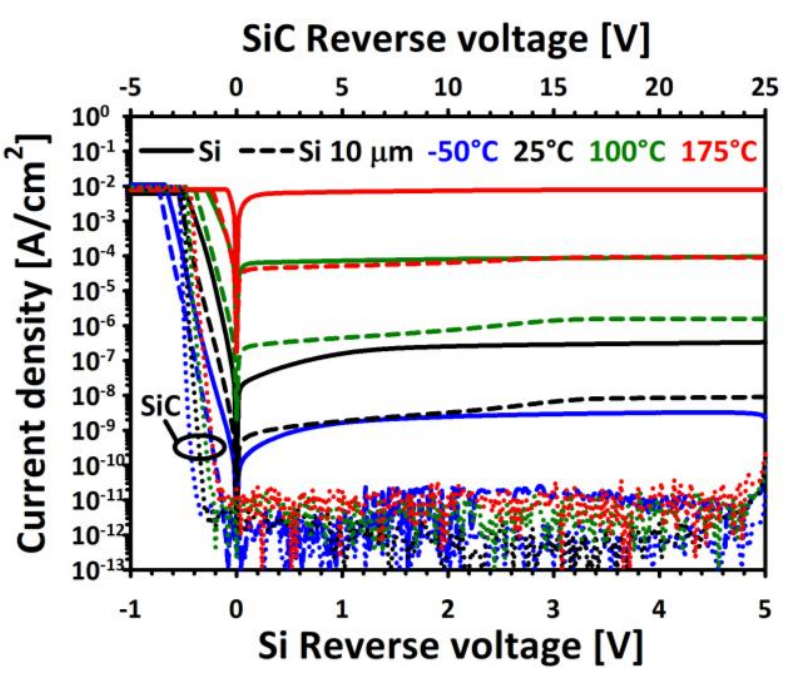

(a)

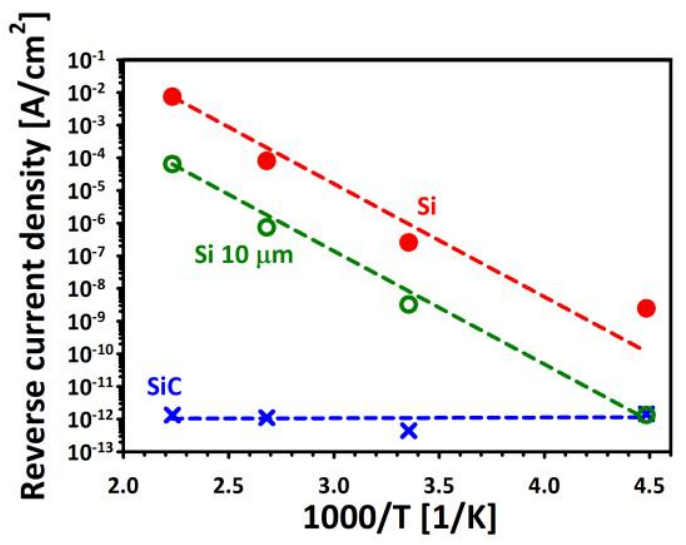

(b)

Figure 4. (a) Diode current-voltage characteristics and (b) reverse current density as a function of temperature for bulk $\mathrm{Si}, 10 \mu \mathrm{m}$-thick $\mathrm{Si}$ and epitaxied $\mathrm{SiC}$ diodes measured at four different temperatures $\left(-50^{\circ} \mathrm{C}, 25^{\circ} \mathrm{C}, 100{ }^{\circ} \mathrm{C}\right.$ and $\left.175^{\circ} \mathrm{C}\right)$. Diode area is $0.09 \mathrm{~cm}^{2}$.

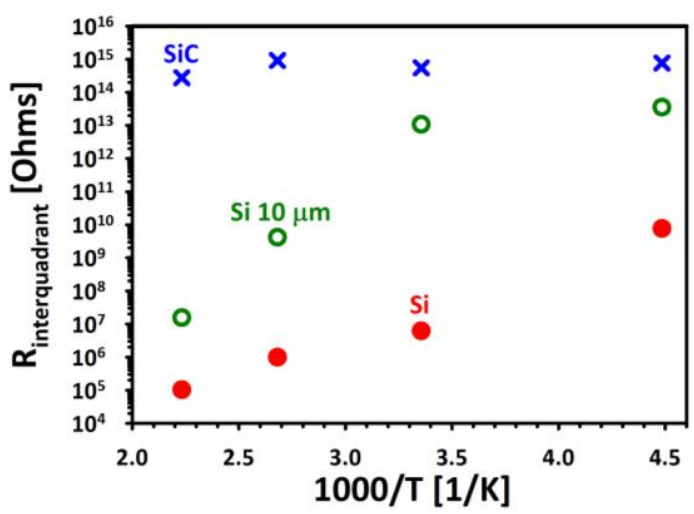

Figure 5. $\mathrm{R}_{\text {interquadrant }}$ versus measuring temperature for different substrate four-quadrant diodes.

In order to investigate possible temperature effects on interquadrant resistance of the segmented diodes, electrical measurements on four-quadrant devices fabricated on the different semiconductor substrates were also carried out at different temperatures. Three independent HP 4155B source monitor units (SMUs) were connected to the first quadrant, to the second, third and fourth quadrants shorted together and to the backside terminal. Current versus voltage curves were measured by applying a limited $\mathrm{V}_{2}$ voltage sweep (from $-1 \mathrm{~V}$ to $+1 \mathrm{~V}$ and $-5 \mathrm{~V}$ to $+5 \mathrm{~V}$ for $\mathrm{Si}$ and $\mathrm{SiC}$ devices, respectively) to the three connected neighbour quadrants while measuring the $I_{1}$ current in the other quadrant that was kept at zero potential. These curves were measured for several diode backside reverse voltages. An estimation of interquadrant resistance was obtained from the slope of $I_{1}$ versus $V_{2}$ characteristics $\left(R_{\text {interquadrant }}=1 /\left|\left(\mathrm{dI}_{1} / \mathrm{dV}_{2}\right)\right|\right)$. The slopes were obtained by means of linear curve fits of the measured characteristics at the various diode backside reverse biases.

Figure 5 shows $\mathrm{R}_{\text {interquadrant }}$ as a function of measuring temperature for a set of bulk $\mathrm{Si}, 10$ $\mu \mathrm{m}$-thick Si and epitaxied SiC four-quadrant devices (4Q-A type). The interquadrant resistance values correspond to high substrate reverse bias conditions (100 V for bulk Si and epitaxied $\mathrm{SiC}$ 
diodes and $10 \mathrm{~V}$ for $10 \mu \mathrm{m}$-thick $\mathrm{Si}$ devices). From the figure, lower $\mathrm{R}_{\text {interquadrant }}$ values are clearly extracted for bulk silicon devices when increasing the measurement temperature. Although higher values are obtained for the diodes fabricated on the $10 \mu \mathrm{m}$-thick silicon membranes, $R_{\text {interquadrant }}$ temperature dependence is also observed for these thinner Si devices. The highest $\mathrm{R}_{\text {interquadrant }}$ values are clearly obtained for the $\mathrm{SiC}$ four-quadrant diodes, with no appreciable temperature dependence for the studied conditions.

\subsection{Visible light effects}

Due to the larger $\mathrm{SiC} 4 \mathrm{H}$ bandgap energy $(3.2 \mathrm{eV})$ compared to $\mathrm{Si}(1.1 \mathrm{eV})$, visible light, with wavelengths in the range of $400 \mathrm{~nm}$ to $700 \mathrm{~nm}$ and corresponding photon energies between 3.1 $\mathrm{eV}$ and $1.65 \mathrm{eV}$, is not expected to generate electron-hole pairs in the $\mathrm{SiC}$ material. Owing to this lower susceptibility to visible light, which may be present in real operation applications, there is interest in $\mathrm{SiC}$ devices, which could simplify some of the experiments.

In order to get a rough idea about the impact of visible light conditions on the electrical characteristics of the devices fabricated on the different substrates, the illumination system of the wafer prober microscope was used. While performing the electrical measurements, four different visible light conditions for the set-up were considered: "dark", "ambient", "minimum" and "maximum" illumination. In the "dark" condition the device under test (DUT) was measured in the light-proof wafer prober microchamber (using a metal piece to prevent any light entering through the microscope glass window). In the "ambient" condition, the metal piece was not used, so that room ambient light was allowed to illuminate the DUT. Under the "minimum" and "maximum" illumination conditions, the LEDs of the microscope illumination system were switched on, so that additional light than in the "ambient" condition reached the DUT. It has to be commented here that no calibrated regulation was available for the LEDs illumination system and this could have affected especially the "minimum" light condition (in some cases the LEDs could have been a bit more or less active). On the other hand, the "maximum" illumination condition is thought to be more precise, as it corresponded to the maximum illumination power of the system.

Figure 6(a) shows reverse current for bulk Si, $10 \mu$ m-thick Si and epitaxied $\mathrm{SiC}$ diodes exposed to the different visible light conditions. To better allow illumination, the data was taken for single diodes with a $0.09 \mathrm{~cm}^{2}$ area and without metal layer on most of the diode, only a metal ring around it. From Figure 6(a) a clear increase in reverse current levels is observed for the bulk Si and 10- $\mu \mathrm{m}$ thick Si devices when increasing the visible light illumination conditions. In contrast, no significant visible light dependence is observed for the $\mathrm{SiC}$ diodes reverse current under the studied illumination conditions.

In order to investigate possible visible light effects on the interquadrant resistance of the segmented diodes, electrical measurements on four-quadrant devices fabricated on the different semiconductor substrates were also carried out. Four-quadrant diodes (4Q-C type) without metal layer on most of the diode quadrants were used for these measurements. Figure 6(b) shows the obtained $R_{\text {interquadrant }}$ results as a function of illumination condition. From the figure, lower $\mathrm{R}_{\text {interquadrant }}$ values are clearly obtained for bulk silicon and $10-\mu \mathrm{m}$ thick $\mathrm{Si}$ when increasing the visible light illumination. The highest $\mathrm{R}_{\text {interquadrant }}$ values are clearly obtained for the $\mathrm{SiC}$ four-quadrant diodes, with no significant visible light dependence under the studied illumination conditions. 


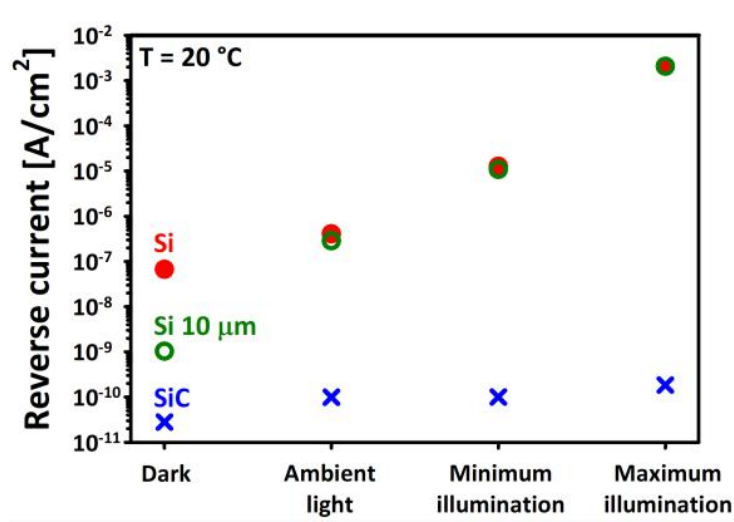

(a)

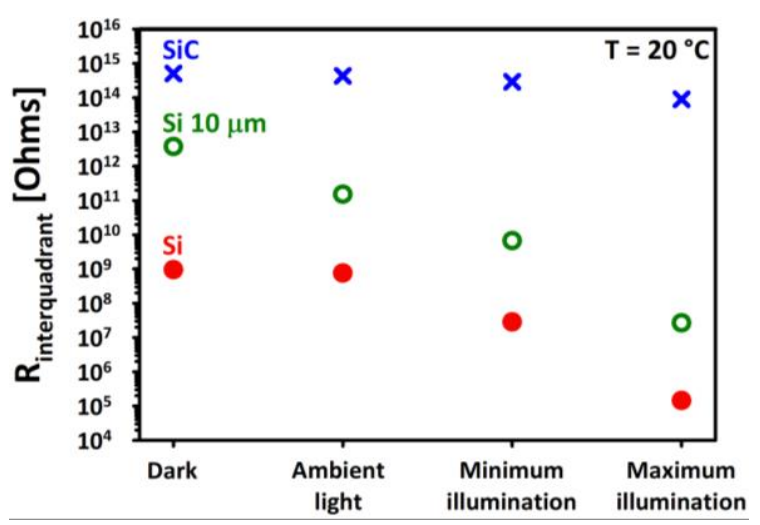

(b)

Figure 6. (a) Diode reverse current density and (b) Extracted $\mathrm{R}_{\text {interquadrant }}$ for bulk Si, $10 \mu \mathrm{m}$ thick $\mathrm{Si}$ and epitaxied $\mathrm{SiC}$ diodes exposed to different visible light conditions.

\section{Electron irradiation effects}

In order to investigate the radiation hardness of the devices fabricated on the different substrates, some samples were subjected to unbiased $2 \mathrm{MeV}$ electron irradiations (the terminals of the devices were left floating) at room temperature. The irradiations were carried out using the electron accelerator at Takasaki-JAEA in Japan for three different independent fluences $(\phi=$ $1 \times 10^{14} \mathrm{e} / \mathrm{cm}^{2}, 1 \times 10^{15} \mathrm{e} / \mathrm{cm}^{2}$ and $1 \times 10^{16} \mathrm{e} / \mathrm{cm}^{2}$, with corresponding total ionizing doses about 2.5 $\operatorname{Mrad}(\mathrm{Si}), 25 \mathrm{Mrad}(\mathrm{Si})$ and $250 \mathrm{Mrad}(\mathrm{Si})$ ). After irradiation, the samples were exposed to room temperature during two weeks and no other intentional annealing was performed.

Figure 7(a) shows diode current-voltage characteristics measured for bulk $\mathrm{Si}, 10 \mu \mathrm{m}$-thick $\mathrm{Si}$ and epitaxied $\mathrm{SiC}$ diodes at different $2 \mathrm{MeV}$ electron irradiation fluences. A progressive increase of leakage current in reverse bias operation is observed for increasing e-irradiation fluence. Moreover, some radiation-induced current lowering in forward operation seems to be observed for the more irradiated bulk silicon devices, which could probably be associated to the device series resistance increase. This increasing resistivity may result from changes in the free carrier concentration due to carrier removal by the radiation-induced point defects [16]. For the case of the epitaxied $\mathrm{SiC}$ diodes, relatively low reverse currents are obtained for $1 \times 10^{14} \mathrm{e} / \mathrm{cm}^{2}$ and $1 \times 10^{15} \mathrm{e} / \mathrm{cm}^{2}$ fluences, however, no clear diode characteristics have been observed for $1 \times 10^{16} \mathrm{e} / \mathrm{cm}^{2}$ irradiated devices. This radiation-induced phenomenon in $\mathrm{SiC}$ devices needs to be further studied. In particular, device performance as a detector remains to be checked and the results from recent proton and neutron irradiations could shed some further light on the performance of the irradiated $\mathrm{SiC}$ devices.

Figure 7(b) shows the electron irradiation dependence of leakage current at some fixed reverse bias conditions ( $2 \mathrm{~V}$ for bulk Si and $10 \mu \mathrm{m}$-thick Si devices and $5 \mathrm{~V}$ for epitaxied $\mathrm{SiC}$ diodes). From these results, a value of $4.5 \times 10^{-19} \mathrm{~A} / \mathrm{cm}$ has been extracted for the leakage current damage rate $(\alpha)$ (with $\mathrm{I}_{\mathrm{vol}}=\alpha \cdot \phi$ and $\mathrm{I}_{\mathrm{vol}} \equiv \mathrm{I} /($ area $\mathrm{x}$ depletion depth)). Taking into account a nonionizing energy loss (NIEL) relative hardness factor of $2.49 \times 10^{-2}$ for the $2 \mathrm{MeV}$ electrons with respect to $1 \mathrm{MeV}$ neutrons [17], the obtained $\alpha$ value is in the range of published results for irradiated silicon [18]. From the $10 \mu \mathrm{m}$-thick Si device results, factors around 1.8, 8 and 50 have been extracted for the radiation-induced diode current degradation, being these somewhat higher than the ones obtained for gamma irradiation at comparable total ionizing doses [3]. 


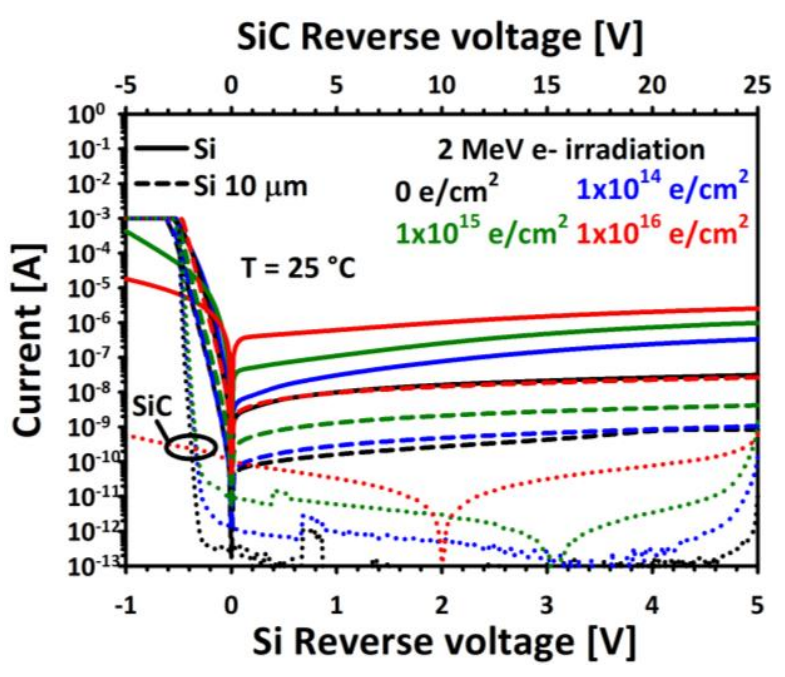

(a)

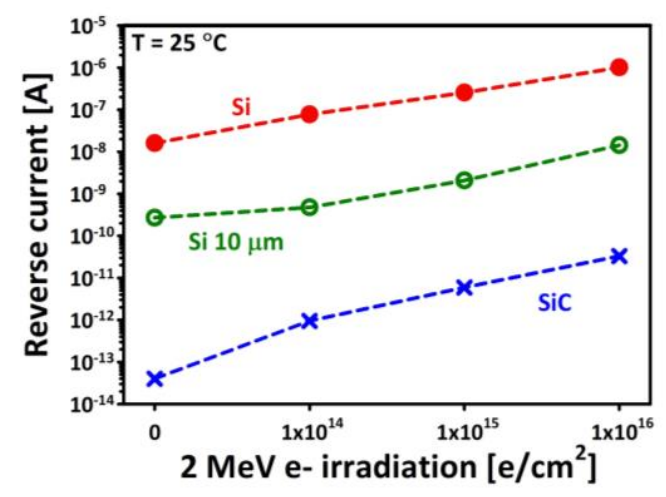

(b)

Figure 7. (a) Diode current-voltage characteristics and (b) reverse current measured for bulk Si, $10 \mu \mathrm{m}$-thick $\mathrm{Si}$ and epitaxied $\mathrm{SiC}$ diodes (4Q-B type) at different $2 \mathrm{MeV}$ electron irradiation fluences.

Figure 8(a) shows interquadrant resistance results as a function of electron fluence. Whereas electron irradiation seems to reduce $\mathrm{R}_{\text {interquadrant }}$ for bulk $\mathrm{Si}$ and epitaxied $\mathrm{SiC}$ devices, no clear trend is observed for the devices fabricated on the $10 \mu \mathrm{m}$ silicon membranes. The previous studies with gamma irradiation on similar thin film devices [3], showed a slight increase of $\mathrm{R}_{\text {interquadrant }}$ after gamma irradiation. $\mathrm{R}_{\text {interquadrant }}$ increase was explained by the presence radiation-induced positive charges in the isolation dielectric, which would lead the ntype silicon surface to a deeper accumulation condition, thus improving interquadrant isolation. For the present case of $2 \mathrm{MeV}$ electron irradiation, a possible explanation could be a trade-off between positive charge trapping in interquadrant isolation and semiconductor surface damage. In fact, the results from irradiated four-quadrant diodes with higher interquadrant distance (4QC instead of 4Q-B type devices from Figure 8) show a slight $\mathrm{R}_{\text {interquadrant }}$ decrease with electron irradiation.

In order to evaluate radiation-induced charge build-up in the diode interquadrant isolation dielectric, several MOS capacitors that use the same isolation oxide as gate dielectric were also irradiated. A clear stretch-out and radiation-induced shift of $\mathrm{C}-\mathrm{V}$ curves towards negative gate voltages was observed for the irradiated MOS capacitors on the Si substrates. This is indicative of radiation-induced positive charge build-up. The extracted flat band voltage values $\left(\mathrm{V}_{\mathrm{fb}}\right)$ have been compared with the one expected for an ideal MOS structure with $4.25 \mathrm{eV}$ metal work function, corresponding to the aluminium gate electrode. From this, an estimation of the effective trapped charge density $\left(\mathrm{N}_{\text {eff }}\right)$, defined as a fixed charge located at the silicon/dielectric interface, has been obtained. Figure 8(b) shows the extracted $\mathrm{N}_{\text {eff }}$ values from irradiated MOS capacitors. From the figure, low $\mathrm{N}_{\text {eff }}$ values, in the range of $+3.5 \times 10^{10} \mathrm{~cm}^{-2}$ and $-1.4 \times 10^{10} \mathrm{~cm}^{-2}$, have been obtained for non-irradiated Bulk $\mathrm{Si}$ and $10 \mu \mathrm{m}$-thick $\mathrm{Si}$ devices, respectively (assuming theoretical $\mathrm{V}_{\mathrm{fb}}$ values of $-0.16 \mathrm{~V}$ and $-0.32 \mathrm{~V}$ for MOS capacitor on their corresponding n-type silicon substrates). However, positive $\mathrm{N}_{\text {eff }}$ values roughly in the range of $1 \times 10^{12} \mathrm{~cm}^{-2}, 2 \times 10^{12} \mathrm{~cm}^{-2}$ and $3.5 \times 10^{12} \mathrm{~cm}^{-2}$ are obtained for the devices irradiated to $1 \times 10^{14}$ $\mathrm{e} / \mathrm{cm}^{2}, 1 \times 10^{15} \mathrm{e} / \mathrm{cm}^{2}$ and $1 \times 10^{16} \mathrm{e} / \mathrm{cm}^{2}$, respectively. These values are in reasonable agreement 
with previous results obtained on gamma irradiated $10 \mu \mathrm{m}$-thick Si devices [3], when taking into account the equivalent total ionizing dose for the $2 \mathrm{MeV}$ electron irradiations.

For the case of the MOS capacitors on the epitaxied SiC substrate, smaller radiationinduced shift of $\mathrm{C}-\mathrm{V}$ curves was observed after $1 \times 10^{14} \mathrm{e} / \mathrm{cm}^{2}$ and $1 \times 10^{15} \mathrm{e} / \mathrm{cm}^{2}$ irradiation. In principle, this could point to an improved radiation hardness for their $\mathrm{Si}_{3} \mathrm{~N}_{4}$-free interquadrant isolation stack. However, similarly to diode I-V measurements, no functional MOS C-V characteristics could be measured for $1 \times 10^{16} \mathrm{e} / \mathrm{cm}^{2}$ irradiated devices. Further studies would be needed for this radiation-induced phenomenon in SiC devices.

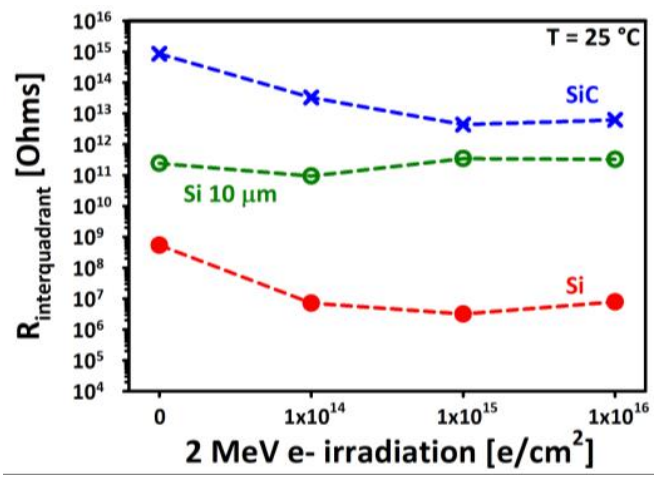

(a)

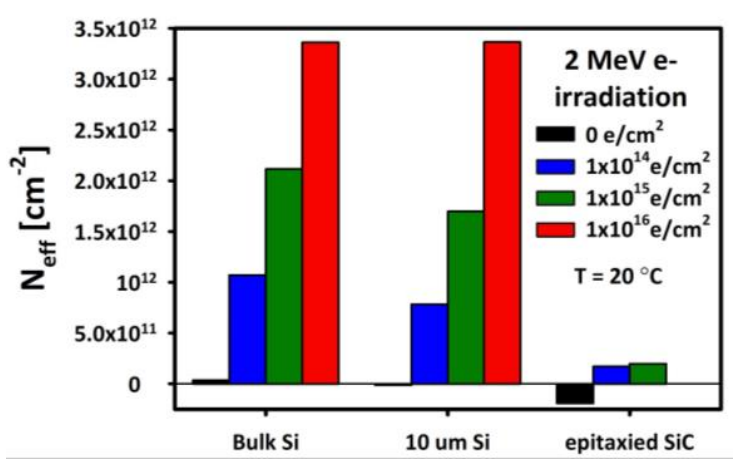

(b)

Figure 8. (a) Extracted $\mathrm{R}_{\text {interquadrant }}$ as a function of $2 \mathrm{MeV}$ electron irradiation fluence for bulk $\mathrm{Si}, 10 \mu \mathrm{m}$-thick $\mathrm{Si}$ and epitaxied $\mathrm{SiC}$ four-quadrant diodes (4Q-B type). (b) effective trapped charge densities $\left(\mathrm{N}_{\text {eff }}\right)$ in the isolation dielectric extracted from $\mathrm{C}-\mathrm{V}$ curves measured at $1 \mathrm{kHz}$ on electron irradiated MOS capacitors with area $8.17 \times 10^{-3} \mathrm{~cm}^{2}$.

\section{Summary}

Segmented four-quadrants diodes, intended for X-ray beam alignment, as well as other potential space or astronomy applications, have been fabricated on ultrathin $(10 \mu \mathrm{m})$ and bulk Si, as well as on epitaxied SiC substrates. The improved performance shown by silicon carbide devices at variable temperatures and visible light illumination conditions could simplify some applications in which silicon devices are currently used. However, the radiation-induced behaviour at high irradiation fluences, as well as further $\mathrm{SiC}$ technology developments to process on high resistive (semi-insulating) bulk SiC substrates, need to be further studied.

\section{Acknowledgments}

This work has been partially financed by Spanish Ministry of Education and Science through the Particle Physics National Program FPA2015-69260-C3-3-R (MINECO/FEDER UE) and the EU Project Advanced European Infrastructures for Detectors and Accelerators (AIDA-2020INFRAIA-2015). This work has made use of the Spanish ICTS Network MICRONANOFABS partially supported by MINECO. Catalan Institute of Nanocience and Nanotechnology (ICN2) and X. Borrisé are acknowledged for the FIB, SEM and EDX inspections. 


\section{References}

[1] M.R. Fuchs, K. Holldack, M. Bullough, et al., Transmissive x-ray beam position monitors with submicron position- and submillisecond time resolution, Rev. Sci. Instrum. 79 (2008) 063103.

[2] C. Cruz, G. Jover-Manas, O. Matilla, et al., $10 \mu \mathrm{m}$ thin transmissive photodiode produced by ALBA Synchrotron and IMB-CNM-CSIC, J. Instrum. 10 (2015) C03005.

[3] J.M. Rafí, G. Pellegrini, D. Quirion, et al., $10 \mu m$-thick four-quadrant transmissive silicon photodiodes for beam position monitor application: electrical characterization and gamma irradiation effects, J. Instrum. 12 (2017) C01004.

[4] P. Roth, A. Georgiev and H. Boudinov, Design and construction of a system for sun-tracking, Renew. Energ. 29 (2004) 393.

[5] F. Nava, G. Bertuccio, A. Cavallini, et al., Silicon carbide and its use as a radiation detector material, Meas. Sci. Technol. 19 (2008) 102001.

[6] P. Godignon, X. Jordà, M. Vellvehi, et al., SiC Schottky Diodes for Harsh Environment Space Applications, IEEE Trans. Ind. Electron. 58 (2011) 2582.

[7] A.L. Barry, B. Lehmann, D. Fritsch, D. Bräunig, Energy dependence of electron damage and displacement threshold energy in 6H silicon carbide, IEEE Trans. Nucl. Sci. 38 (1991) 1111.

[8] C. Claeys, E. Simoen. Radiation effects in advanced semiconductor materials and devices, Berlin Heidelberg: Springer; 2002.

[9] M. Moll, Development of radiation hard sensors for very high luminosity colliders - CERN RD50 project, Nucl. Instrum. Meth. A 511 (2003) 97.

[10] A.A. Lebedev, K.S. Davydovskaya, A.M. Strelchuck, et al., Radiation resistance of 4H-SiC Schottky diodes under irradiation with 0.9-MeV electrons, J. Surf. Investig.-X-Ra. 11 (2017) 924.

[11] J. Morse, B. Solar, H. Graafsma, Diamond X-ray beam-position monitoring using signal readout at the synchrotron radiofrequency, J. Synchrotron Radiat. 17 (2010) 456.

[12] R. Klanner, E. Fretwurst, I. Pintilie, J. Schwandt, J. Zhang, Study of high-dose X-ray radiation damage of silicon sensors, Nucl. Instrum. Meth. A 732 (2013) 117.

[13] M. Lozano, G. Pellegrini, C. Fleta, et al., Comparison of Radiation Hardness of P-in- $N, N$-in- $N$, and N-in-P Silicon Pad Detectors, IEEE Trans. Nucl. Sci. 52 (2005) 1468.

[14] L. Andricek, G. Lutz, M. Reiche, et al., Processing of ultra-thin silicon sensors for future $e^{+} e^{-}$ linear collider experiments, IEEE Trans. Nucl. Sci. 51 (2004) 1655.

[15] A. Czerwinski, E. Simoen, A. Poyai, C. Claeys, Activation energy analysis as a tool for extraction and investigation of p-n junction leakage current components, J. Appl. Phys. 94 (2003) 1218.

[16] H.J. Stein, R. Gereth, Introduction Rates of Electrically Active Defects in n- and p-Type Silicon by Electron and Neutron Irradiation, J. Appl. Phys. 39 (1968) 2890.

[17] G.P. Summers, E.A. Burke, Damage correlations in semiconductors exposed to gamma, electron and proton radiations, IEEE Trans. Nucl. Sci. 40 (1993) 1372.

[18] G. Lindström, et al., Radiation hard silicon detectors - Developments by the RD48 (ROSE) collaboration, Nucl. Instrum. Meth. A 466 (2001) 308. 